\title{
THE CAPACITY METRIC ON RIEMANN SURFACES
}

\author{
C. DAVID MINDA
}

\section{Introduction}

Let $c_{\beta}(\zeta)|d \zeta|$ denote the capacity metric on a Riemann surface. In this paper two basic facts dealing with this metric are established. First, if $f: X \rightarrow Y$ is an analytic mapping of Riemann surfaces, then $f$ is distance decreasing relative to the capacity metric. For $X, Y \notin O_{G}$, a necessary and sufficient condition that $f: X \rightarrow Y$ be an isometry is given: $f$ must be injective and $Y \backslash f(X)$ must be a closed set of capacity zero. This condition is obtained from an analogous property of the Green's function. Let $g_{X}, g_{Y}$ denote the Green's function on $X, Y$, respectively. If $g_{X}=g_{Y} \circ f$, then $f$ must be as before. The second property of the capacity metric that we derive is an interpretation of this metric in terms of the reduced modulus of a path family of cycles homologous to a point. This naturally leads to the question of an analogous interpretation of other metrics. This question is answered for the Hahn metric by using the family of closed curves homotopic to a point.

\section{Definition of the capacity metric}

Let $X$ be a Riemann surface. The customary notation for the capacity metric on $X$ is $c_{\beta}(\zeta)|d \zeta|$, where $\beta$ represents the ideal boundary of $X$. This notation is not convenient because we have need to consider the capacity metric on several surfaces simultaneously. In order to clearly indicate the dependence on the surface $X$, we shall employ the notation $c_{X}(\zeta)|d \zeta|$ for the capacity metric on $X$. Now we define the capacity metric. Suppose $\zeta \in X$ and $t$ is a local parameter in a neighborhood of $\zeta$ such that $t(\zeta)=0$. Let $\mathscr{A}_{\zeta}^{*}(X)$ denote the family of all multiplevalued analytic functions $F$ defined on $X$ such that $|F|$ is single-valued, $F(\zeta)=0$ and $F^{\prime}(\zeta)=1$ for one of the branches. Here $F^{\prime}(\zeta)$ represents the derivative of $F_{0} t^{-1}$ at the origin. Set

Then ([7], [8, pp. 177-178])

$$
M[F]=\sup _{z \in X}|F(z)| .
$$

$$
\frac{1}{c_{X}(\zeta)}=\min _{F \in \mathscr{A}_{\zeta}^{*}(X)} M[F]
$$


If $c_{X}(\zeta)>0$, then the unique minimizing function is $F_{X}=\exp \left(p_{X}+i p_{X}^{*}\right)$, where $p_{X}$ is the capacity function with pole at $\zeta$. The usual notation for this capacity function is $p_{\beta}$, but we need to indicate the dependence on $X$.

The condition $c_{X}(\zeta)=0$ is independent of the point $\zeta \in X[8, \mathrm{p} .178]$. The identical vanishing of the capacity metric $c_{X}(\zeta)|d \zeta|$ is equivalent to $X \in O_{G}$ [7]. Recall that $O_{G}$ is the class of Riemann surfaces which do not possess a Green's function. For $c_{X}(\zeta)>0$,

$$
p_{X}(z, \zeta)=k_{X}(\zeta)-g_{X}(z, \zeta),
$$

where $g_{X}(z, \zeta)$ is the Green's function for $X$ with logarithmic singularity at $\zeta$ and $k_{X}(\zeta)=k_{\beta}(\zeta)$ is the Robin constant defined by

$$
k_{X}(\zeta)=\lim _{t \rightarrow 0}\left[g_{X}(z, \zeta)+\log |t|\right]
$$

[8, p. 55]. Thus, if $c_{X}(\zeta)>0$, then

$$
\left|F_{X}\right|=\exp \left(p_{X}\right) \leqq k_{X}(\zeta)
$$

and $M\left[F_{X}\right]=k_{X}(\zeta)$. Consequently,

$$
c_{X}(\zeta)=\exp \left(-k_{X}(\zeta)\right) .
$$

If $X$ is a hyperbolic simply connected Riemann surface, then it is elementary to show that $c_{X}(\zeta)|d \zeta|=\lambda_{X}(\zeta)|d \zeta|$, where $\lambda_{X}(\zeta)|d \zeta|$ denotes the hyperbolic metric on $X$ with constant curvature -4 . An explicit formula for the capacity metric of an annulus is given in [9].

\section{Green's function}

In the nontrivial case the definition of the capacity metric involves the Green's function and the Robin constant. In this section we derive a property of the Green's function that will be basic in establishing a result for the capacity metric in Section 4. Moreover, this property of the Green's function might be of independent interest.

We begin by recalling a precise form of the Lindelöf principle that was established by Heins [2]. Suppose $X, Y \notin O_{G}$ and $f: X \rightarrow Y$ is an analytic function. Then for any $\omega \in Y$,

$$
g_{Y}(f(z), \omega)=\sum_{f(\zeta)=\omega} n(\zeta, f) g_{X}(z, \zeta)+u_{\omega}(z),
$$

where $u_{\omega}$ is a nonnegative harmonic function on $X$ and $n(\zeta, f)$ is the order of $f$ at the point $\zeta$. Furthermore, $u_{\omega}$ has the canonical decomposition $u_{\omega}=q_{\omega}+s_{\omega}$, where $q_{\omega}$ is quasibounded and $s_{\omega}$ is singular. The following dichotomy holds: either $q_{\omega}>0$ for all $\omega \in Y$ or else $q_{\omega}=0$ for all $\omega \in Y$. The function $f$ is said to belong to the class $B l$ if $q_{\omega}=0$ for all $\omega \in Y$. Also, $s_{\omega}=0$ except possibly for a set of $\omega$ of capacity 
zero. If $u_{\omega}=0$ for all $\omega \in Y$, then $f$ is said to be of type $B l_{1}$. Set

$$
v_{f}(w)=\sum_{f(z)=w} n(z, f) .
$$

If $f$ is of type $B l$, then either $v=\sup \left\{v_{f}(w): w \in Y\right\}$ is finite and $\left\{w: v_{f}(w)<v\right\}$ is a closed set of capacity zero or else $\left\{w: v_{f}(w)<\infty\right\}$ is an $F_{\sigma}$ set of capacity zero. Thus $f$ covers $Y$ exactly the same number of times (possibly infinite) except for an $F_{\sigma}$ set of capacity zero. If the valence of $f$ is finite, then the exceptional set is closed. If $f$ is of type $B l_{1}$, then $v_{f}(w)$ is constant (possibly infinite).

Recall that the Green's function is a conformal invariant. This means that if $X, Y \notin O_{G}$ and $f: X \rightarrow Y$ is a conformal mapping, then $g_{Y}(f(z), f(\zeta))=g_{X}(z, \zeta)$ for all $(z, \zeta) \in X \times X$. The following theorem is sort of a converse.

Theorem 1. Suppose $X, Y \notin O_{G}$ and $f: X \rightarrow Y$ is an analytic function. If there exist distinct points, $p, q \in X$ such that

$$
g_{Y}(f(p), f(q))=g_{X}(p, q),
$$

then $f$ is injective and $Y \backslash f(X)$ is a closed set of capacity zero.

Proof. We begin by showing that if (1) holds, then $f(\zeta) \neq f(q)$ for all $\zeta \in X \backslash\{q\}$, $n(q, f)=1$ and $g_{Y}(f(\zeta), f(q))=g_{X}(\zeta, q)$ for all $\zeta \in X$. The sharp form of the Lindelöf principle gives

$$
g_{Y}(f(\zeta), f(q))=\sum_{f(z)=f(q)} n(z, f) g_{X}(\zeta, z)+u(\zeta)
$$

for $\zeta \in X$, where $u=u_{f(q)}$ is a nonnegative harmonic function. For $\zeta=p$ equation (2) yields

$$
\begin{aligned}
g_{Y}(f(p), f(q)) & =\sum_{f(z)=f(q)} n(z, f) g_{X}(p, z)+u(p) \\
& \geqq n(q, f) g_{X}(p, q)+u(p) \\
& \geqq g_{X}(p, q) .
\end{aligned}
$$

Equation (1) implies that equality holds throughout, so $n(q, f)=1, u=0$ and $f(\zeta) \neq$ $f(q)$ for all $\zeta \in X \backslash\{q\}$. Thus, equation (2) becomes

$$
g_{Y}(f(\zeta), f(q))=g_{X}(\zeta, q)
$$

for all $\zeta \in X$. This establishes the claim made at the beginning of the paragraph. Now, we complete the proof. From (3) and the symmetry of the Green's function, we obtain $g_{Y}(f(q), f(\zeta))=g_{X}(q, \zeta)$ for all $\zeta \in X$. The argument given in the preceding paragraph immediately implies that $f(z) \neq f(\zeta)$ for all $z \in X \backslash\{\zeta\}$, $n(z, f)=1$ and

$$
g_{Y}(f(z), f(\zeta))=g_{X}(z, \zeta)
$$

for all $z \in X$. But this also holds for all $\zeta \in X$. It follows directly that $f$ is injective. The remainder of the theorem is obtained from the sharp form of the Lindelö principle. From equation (4) we conclude that $u_{\omega}=0$ for all $\omega \in f(X)$. In particular, 
$q_{\omega}=0$ for all $\omega \in f(X)$. The dichotomy given in the Lindelöf principle implies that we actually have $q_{\omega}=0$ for all $\omega \in Y$. Hence, $f$ is of type $B l$. Because $f$ is injective, $\sup \left\{v_{f}(w): w \in Y\right\}=1$. Then $Y \backslash f(X)=\left\{w \in Y: v_{f}(w)<1\right\}$ is a closed set of capacity zero.

It is elementary to show that if $f: X \rightarrow Y$ is injective and $Y \backslash f(X)$ has capacity zero, then equality holds in (1) for all $p, q \in X$. Just note that $Y$ and $Y \backslash f(X)$ possess the same Green's function in this situation.

\section{Properties of the capacity metric}

First, we establish the elementary result that the hyperbolic metric dominates the capacity metric.

Theorem 2. Let $X$ be a hyperbolic Riemann surface. Then $c_{X}(\zeta)|d \zeta| \leqq \lambda_{X}(\zeta)|d \zeta|$. If equality holds at a single point, then $X$ is simply connected.

Proof. There is nothing to prove if $X \in O_{G}$, so we assume $X \notin O_{G}$. Fix $\zeta \in X$ and a local coordinate $t$ at $\zeta$ with $t(\zeta)=0$. Suppose $F_{X} \in \mathscr{A}_{\zeta}^{*}(X)$ is the unique extremal function relative to the local coordinate $t$. Let $\mathbf{D}$ denote the open unit disk and $\pi: \mathbf{D} \rightarrow X$ an analytic universal covering such that $\pi(0)=\zeta$. Then $c_{X}(\zeta) F_{X} \circ \pi$ is a single-valued analytic mapping of $\mathbf{D}$ into itself that fixes the origin. Also, this function vanishes at each point of the set $\pi^{-1}(\zeta)$. Schwarz' lemma gives

$$
c_{X}(\zeta)\left|F_{X}^{\prime}(\zeta)\right|\left|\pi^{\prime}(0)\right| \leqq 1,
$$

where $\pi^{\prime}(0)$ denotes the derivative of $t \circ \pi$ at the origin. Thus,

$$
c_{X}(\zeta) \leqq 1 /\left|\pi^{\prime}(0)\right|=\lambda_{X}(\zeta) .
$$

Equality implies that $c_{X}(\zeta) F_{X} \circ \pi$ is a rotation of $\mathbf{D}$ about the origin. In particular, it vanishes just once, so $\pi^{-1}(\zeta)=\{0\}$. This implies that $\pi$ is univalent, so $X$ must be simply connected.

Next, we demonstrate that an analytic function is distance decreasing relative to the capacity metric.

Theorem 3. Suppose $X$ and $Y$ are Riemann surfaces and $f: X \rightarrow Y$ is an analytic function. Then

$$
f^{*}\left(c_{Y}(\zeta)|d \zeta|\right) \leqq c_{X}(\zeta)|d \zeta|,
$$

where $f^{*}\left(c_{Y}(\zeta)|d \zeta|\right)$ denotes the pull-back to $X$ via $f$ of the capacity metric on $Y$. If $X \notin O_{G}$ and equality holds at a point, then $f$ is injective and $Y \backslash f(X)$ is a closed set of capacity zero.

Proof. Fix $\zeta \in X$ and set $\omega=f(\zeta)$. Let $u$ be a local coordinate at $\omega$ with $u(\omega)=0$. If $n(\zeta, f) \geqq 2$, then the pull-back of any metric via $f$ vanishes at $\zeta$. There is nothing to prove in this case, so we may assume that $n(\zeta, f)=1$. In this situation $f$ is univalent 
in a neighborhood of $\zeta$ so that $t=u \circ f$ is a local coordinate at $\zeta$ with $t(\zeta)=0$. Let $f^{\prime}(\zeta)$ denote the derivative of $u \circ f \circ t^{-1}$ at the origin. Again, there is nothing to prove if $Y \in O_{G}$, so we assume $c_{Y}(\omega)>0$. Let $F_{Y} \in \mathscr{A}_{\omega}^{*}(Y)$ be the unique extremal for $c_{Y}(\omega)$ relative to the local coordinate $u$. Then $\left(F_{Y} \circ f\right) / f^{\prime}(\zeta) \in A_{\zeta}^{*}(X)$ so that

or

$$
\frac{1}{c_{X}(\zeta)} \leqq M\left[\left(F_{Y} \circ f\right) / f^{\prime}(\zeta)\right] \leqq \frac{M\left[F_{Y}\right]}{\left|f^{\prime}(\zeta)\right|}=\frac{1}{c_{Y}(\omega)\left|f^{\prime}(\zeta)\right|}
$$

$$
c_{Y}(\omega)\left|f^{\prime}(\zeta)\right| \leqq c_{X}(\zeta) .
$$

This establishes the inequality in the theorem.

Next, assume that $X \notin O_{G}$ and that equality holds at $\zeta$. Then $Y \notin O_{G}$ and $f^{\prime}(\zeta) \neq 0$. Since equality holds at $\zeta$,

or

$$
\exp \left(-k_{\mathbf{Y}}(\omega)\right)\left|f^{\prime}(\zeta)\right|=\exp \left(-k_{X}(\zeta)\right)
$$

$$
k_{Y}(\omega)-\log \left|f^{\prime}(\zeta)\right|=k_{X}(\zeta) .
$$

Also, when equality holds at $\zeta$ the work in the preceding paragraph shows that $\left(F_{Y} \circ f\right) \mid f^{\prime}(\zeta) \in A_{\zeta}^{*}(X)$ is a minimizing function, so it equals $F_{X}$. This gives $p_{X}=$ $p_{Y} \circ f-\log \left|f^{\prime}(\zeta)\right|$, or

$$
\begin{gathered}
k_{X}(\zeta)-g_{X}(z, \zeta)=k_{Y}(\omega)-g_{Y}(f(z), f(\zeta))-\log \left|f^{\prime}(\zeta)\right|, \\
g_{X}(z, \zeta)=g_{Y}(f(z), f(\zeta)) .
\end{gathered}
$$

By applying Theorem 1, we obtain the desired conclusion.

Observe that if $X \in O_{G}$ and $Y \notin O_{G}$, then Theorem 3 implies that every analytic function $f: X \rightarrow Y$ must be constant. Also, if $X \notin O_{G}, f: X \rightarrow Y$ is injective and $Y \backslash f(X)$ is a closed set of capacity zero, then it is not difficult to show that equality holds in Theorem 3 at every point of $X$.

\section{Reduced modulus interpretation of the capacity metric}

We start by defining the reduced modulus of a special type of family of paths on a Riemann surface that always leads to a metric on the surface.

Let $X$ be a Riemann surface and $\mathscr{F}$ a family of paths on $X$. Suppose $\zeta \in X$ and $t$ is a local coordinate at $\zeta$ such that $t(\zeta)=0$. Assume that the range of this local coordinate contains the disk of radius $R$ centered at the origin. For $0<r<s<R$ assume that the family $\mathscr{A}(r, s)$ of closed Jordan curves in $A(r, s)=\{z \in X: r<|t(z)|<s\}$ which separate the boundary components is a subset of $\mathscr{F}$. The symbol $\mathscr{F}_{\zeta}(r)$ denotes the set of paths in $\mathscr{F}$ which lie in $X \backslash\{z \in X:|t(z)|<r\}$. We show that $M\left(\mathscr{F}_{\zeta}(r)\right)+(1 / 2 \pi) \log r$ increases as $r$ decreases, where $M\left(\mathscr{F}_{\zeta}(r)\right)$ denotes the modulus of the path family $\mathscr{F}_{\xi}(r)$. Note that $\mathscr{F}_{\zeta}(s) \cup \mathscr{A}(r, s) \subset \mathscr{F}_{\xi}(r)$ for $0<r<$ 
$s<R$. Because the families $\mathscr{F}_{\zeta}(s)$ and $\mathscr{A}(r, s)$ have disjoint support [8, p. 321]

$$
M\left(\mathscr{F}_{\zeta}(s)\right)+M(\mathscr{A}(r, s)) \leqq M\left(\mathscr{F}_{\zeta}(r)\right) .
$$

Now, $M(\mathscr{A}(r, s))=(1 / 2 \pi) \log (s / r)[8$, p. 325], so that

Define

$$
M\left(\mathscr{F}_{\zeta}(s)\right)+\frac{1}{2 \pi} \log s \leqq M\left(\mathscr{F}_{\zeta}(r)\right)+\frac{1}{2 \pi} \log r .
$$

$$
\tilde{M}\left(\mathscr{F}_{\zeta}\right)=\lim _{r \downarrow 0} M\left(\mathscr{F}_{\zeta}(r)\right)+\frac{1}{2 \pi} \log r .
$$

This quantity is called the reduced modulus of the family $\mathscr{F}$ at the point $\zeta$. The value of the reduced modulus does depend on the choice of the local coordinate at $\zeta$. It is not difficult to verify that $\exp \left(-2 \pi \tilde{M}\left(\mathscr{F}_{\zeta}\right)\right)|d \zeta|$ is an invariant form, or metric, on $X$; see [4] for analogous results.

Our goal is to express the capacity metric in terms of the reduced modulus of a path family.

Definition. Let $X$ be a Riemann surface and $\zeta \in X$. A 1-cycle $c$ on $X \backslash\{\zeta\}$ is said to be homologous to $\zeta$ if for every neighborhood $U$ of $\zeta, c$ is homologous to a closed Jordan curve in $U \backslash\{\zeta\}$ which winds around $\zeta$ once in the positive direction. Let $\mathscr{H}_{\zeta}$ denote the family of all 1-cycles on $X \backslash\{\zeta\}$ that are homologous to $\zeta$.

Theorem 4. Let $X \ddagger O_{G}$. Then

$$
c_{X}(\zeta)|d \zeta|=\exp \left(-2 \pi \tilde{M}\left(\mathscr{H}_{\zeta}\right)\right)|d \zeta| .
$$

Proof. Fix $\zeta \in X$. It suffices to demonstrate equality at $\zeta$. In fact, it is enough to show equality relative to some fixed local coordinate at $\zeta$. We begin by selecting a local coordinate that will make it easy to demonstrate equality. Let $g(z)=g_{X}(z, \zeta)$ be the Green's function for $X$ with logarithmic singularity at $\zeta$. In a deleted neighborhood of $\zeta$ let $g^{*}$ denote a harmonic conjugate for $g$. Of course, $g^{*}$ is not singlevalued; it is only determined up to an additive multiple of $2 \pi$. However, in a small neighborhood of $\zeta$ the function $t(z)=\exp \left(-g(z)-i g^{*}(z)\right)$ is a local coordinate that satisfies $t(\zeta)=0$. We shall establish equality in terms of this special local coordinate at $\zeta$.

Next, assume that $X$ is the interior of a compact bordered Riemann surface $\bar{X}$. Let $B(\zeta, r)=\{z \in X:|t(z)|<r\}$, where $r>0$ is sufficiently small. Assume that $\partial B(\zeta, r)$ is positively oriented. Then $g$ is harmonic on $Y=X \backslash \overline{B(\zeta, r)}$, has the constant value 0 on $\partial X$ and the constant value $\log (1 / r)$ on $\partial B(\zeta, r)$. Thus, $\omega=g / \log (1 / r)$ is the harmonic measure of $\partial B(\zeta, r)$ with respect to the surface $Y$. It follows that $d \omega$ is the $\Gamma_{h}$-reproducing differential for any 1-cycle $c$ on $Y$ which is homologous to 
$\partial B(q, r)[6$, p. 135]. That is,

$$
\int_{c} \sigma=(\sigma, d \omega)_{Y}=\iint_{Y} \sigma \wedge * d \omega
$$

for any square integrable harmonic differential $\sigma$ on $Y$, where $c$ is as above. The set of such 1-cycles is simply $\mathscr{H}_{\zeta}(r)$. A result of Accola [1] implies that

$$
M\left(\mathscr{H}_{\zeta}(r)\right)=\|d \omega\|_{\mathbf{Y}}{ }^{2} .
$$

By making use of Stokes' theorem, we find that

$$
\begin{gathered}
\|d \omega\|_{Y}^{2}=\iint_{Y}\left(\omega_{x}^{2}+\omega_{y}^{2}\right) d x d y=\iint_{\partial Y} \omega^{*} d \omega \\
=-\int_{\partial B(\zeta, r)}{ }^{*} d \omega=\frac{2 \pi}{\log (1 / r)} .
\end{gathered}
$$

Consequently, $M\left(\mathscr{H}_{\zeta}(r)\right)=-(1 / 2 \pi) \log r$, so that $\tilde{M}_{\zeta}\left(\mathscr{H}_{\zeta}\right)=0$ and

$$
\exp \left(-2 \pi \tilde{M}\left(\mathscr{H}_{\zeta}\right)\right)=1 \text {. }
$$

All that remains is to show that the capacity metric at $\zeta$ also has the value 1 relative to the local coordinate $t$. Now,

$$
k_{X}(\zeta)=\lim _{t \rightarrow 0}(g(z, \zeta)+\log |t|)=0
$$

since $g(z, \zeta)=-\log |t|$, where $t=t(z)$. Thus, $c_{X}(\zeta)=\exp \left(-k_{X}(\zeta)\right)=1$. This completes the proof in case $X$ is the interior of a compact bordered Riemann surface.

The general case follows by making use of an exhaustion of $X$ by compact bordered surfaces. This method has been employed frequently, see [4] and other references mentioned there. For this reason we omit all details in the general case.

\section{Reduced modulus interpretation of other metrics}

It is possible to give a similar reduced modulus interpretation of the Hahn metric. For basic properties of this metric on a Riemann surface, see [5].

Definition. Let $X$ be a Riemann surface and $\zeta \in X$. A closed path $c$ on $X \backslash\{p\}$ is said to be homotopic to $\zeta$ if for every neighborhood $U$ of $\zeta, c$ is freely homotopic to a closed Jordan curve in $U \backslash\{\zeta\}$ which winds around $\zeta$ once in the positive direction. Let $\mathscr{K}_{\zeta}$ denote the family of all closed paths on $X \backslash\{\zeta\}$ that are homotopic to $\zeta$.

Theorem 5. Let $X$ be a Riemann surface. Then $S_{X}(\zeta)|d \zeta|=\exp \left(-2 \pi \tilde{M}\left(\mathscr{K}_{\zeta}\right)\right)$, where $S_{X}(\zeta)|d \zeta|$ denotes the Hahn metric on $X$.

Proof. In [5] it was shown that $S_{X}(\zeta)|d \zeta|=\exp \left(-\tilde{M}_{X}(\zeta)|d \zeta|\right)$, where $\tilde{M}_{X}(\zeta)$ denotes the following extremal value. For any hyperbolic simply connected region 
$\Omega$ on $X$ that contains $\zeta, \tilde{M}_{\Omega}(\zeta)$ denotes the reduced modulus of $\Omega$ at $\zeta$. Then

$$
\tilde{M}_{X}(\zeta)=\sup \tilde{M}_{\Omega}(\zeta)
$$

where the supremum is taken over all hyperbolic simply connected regions $\Omega$ on $X$ that contain $\zeta$. In [5] the modulus of the annulus $\left\{w: r_{1}<|w|<r_{2}\right\}$ was taken to be $\log \left(r_{2} / r_{1}\right)$; note the absence of the factor $1 / 2 \pi$ in front of the logarithm. Now, it is known that $\tilde{M}_{X}(\zeta)=2 \pi \tilde{M}\left(\mathscr{K}_{\zeta}\right)$ [3], so Theorem 5 is established.

If $X$ is a hyperbolic Riemann surface, then

$$
c_{X}(\zeta)|d \zeta| \leqq \lambda_{X}(\zeta)|d \zeta| \leqq S_{X}(\zeta)|d \zeta| .
$$

Theorems 4 and 5 give reduced modulus interpretations for both the capacity metric and the Hahn metric. The preceding inequality naturally suggests the following question: Is there a reduced modulus interpretation of the hyperbolic metric? Of course, the same question can be asked for other metrics on a Riemann surface.

\title{
References
}

[1] Accola, R. D. M.: Differentials and extremal length on Riemann surfaces. - Proc. Nat. Acad. Sci. U.S.A. 46, 1960, 540-543.

[2] Heins, M.: On the Lindelöf principle. - Ann. of Math. (2) 61, 1955, 440-473.

[3] Jenkins, J. A.: On the existence of certain general extremal metrics. - Ann. of Math. (2) 66, $1957,440-453$.

[4] MINDA, C. D.: Extremal length and reproducing differentials on Riemann surfaces. - J. Analyse Math. 29, 1976, 154-202.

[5] Minda, C. D.: The Hahn metric on Riemann surfaces. - Kodai Math. J. 6, 1983, 57-69.

[6] Rodin, B., and L. SARIo: Principal functions. - D. Van Nostrand Company, Inc., Princeton, New Jersey-Toronto-London-Melbourne, 1968.

[7] SARIo, L.: Capacity of the boundary and of a boundary component. - Ann. of Math. (2) 59, 1954, 135-144.

[8] SaRIo, L., and K. OIKAwa: Capacity functions. - Die Grundlehren der mathematischen Wissenschaften 149: Springer-Verlag, Berlin-Heidelberg-New York, 1969.

[9] SuItA, N.: On capacities and kernels on Riemann surfaces. - Arch. Rational Mech. Anal. 46, 1972, 212-217.

\author{
University of Cincinnati \\ Department of Mathematical Sciences \\ Cincinnati, Ohio 45221-0025 \\ USA
}

Received 24 June 1985 